\title{
Dynamical Hysteresis Phenomena in Complex Network Traffic
}

\author{
Mao-Bin $\mathrm{Hu}^{1},{ }^{*}$ Xiang Ling ${ }^{1}$, Rui Jiang ${ }^{1}$, Yong-Hong $\mathrm{Wu}^{2}$, and Qing-Song $\mathrm{Wu}^{1}$ \\ ${ }^{1}$ School of Engineering Science, University of Science and Technology of China, Hefei 230026, P.R.China \\ ${ }^{2}$ Department of Mathematics and Statistics, Curtin University of Technology, Perth WA6845, Australia
}

(Dated: March 10, 2009)

\begin{abstract}
In this brief report, the dynamical hysteresis is investigated in constant-density traffic system of scale-free, small-world and lattice networks. With the local searching strategy, hysteresis persists for scale-free networks, while it disappears for small-world networks and lattice grids when the handling ability of nodes is higher than a certain threshold. With the shortest path routing strategy, hysteresis persists for all three networks investigated. When using shortest path strategy, the system is easy to congest, and difficult to recover to free flow state.
\end{abstract}

PACS numbers: $45.70 . \mathrm{Vn}, 89.75 . \mathrm{Hc}, 05.70 . \mathrm{Fh}$

In the past several decades, a large number of works on the traffic dynamics have been carried out for regular and random networks. As large communication networks with scale-free [1] and small world [2,3] properties become increasingly important, the traffic flow on these networks has drawn more and more attention [4-6].

Recent works mainly discussed a constant packet insert rate while allowing the fluctuation of packet number in the system [7]. On the other hand, study of a constant density traffic flow is important for many real traffic systems, including the Internet, urban traffic system [8], and airline network [9]. The constant density traffic began to attract more attention from researchers $[10,11]$. Here we note that in order to consider a constant density traffic, there should be queue length limit for the node. Otherwise one can not get the packet density of the system. One can introduce a finite queue length $L_{i}$ for each node, and define the packet density of the system as $\rho=\frac{N_{p}}{\Sigma L_{i}}$, where $N_{p}$ is the total number of packets in the network.

In this brief report, we carry out detailed study on the traffic dynamics of constant packet density. The hysteresis phenomenon is investigated with a wide variety of network structures and routing strategies. The simulation shows that the hysteresis phenomenon is affected by both the network structures and the navigation strategies. In particular, it is shown that with the shortest path strategy, the system is easy to congest and difficult to recover to free flow state. For local searching strategy, hysteresis persists on scale-free networks, and disappears when the nodes' handling ability is higher than a threshold on small-world networks and lattice grids.

We construct the model under three kinds of networks: scale-free networks, small-world networks and lattice grids. The scale-free network is constructed by the Barabási-Albert model [1] with $m_{0}=m=4$ (with average degree $\langle k\rangle=2 m=$ 8 ) and network size $N=1000$. The small-world network is constructed by adopting the Newman-Watts (NW) model [3], in which new shortcuts are added instead of rewiring old links [2]. The average degree of NW network is controlled by the probability of adding edges. We set network size $N=1000$ and average degree $\langle k\rangle=8$ which is equal to that of scalefree network. In the lattice grids model, each node is con-

*Electronic address: humaobin@ustc.edu.cn nected by its eight nearest neighbors and a periodic boundary condition is adopted. Therefore, the average degree of nodes is also $\langle k\rangle=8$. In simulation, we set the size of lattice as $N=32 \times 32=1024$.

As in other models, all nodes are treated as both hosts and routers for generating and delivering packets. The capacity of each node is controlled by two parameters: (i) its maximum packet queue length $L$, which is proportional to its degree $k$ : $L=\alpha \times k$; (ii) the maximum number of packets it can handle per time step: $C=\beta \times L$. The capacity and delivering ability of a node are limited and vary from node to node, which meets the real condition in most systems. The FIFO (first-in-firstout) queuing discipline is applied at each node. Once a packet arrives at its destination, it is removed from the system.

Two packet routing strategies are considered: (1) the shortest path routing strategy; (2) the local searching strategy [12]. For the first strategy, the packets are routed by the shortest path from origins to destinations. In the local searching strategy, every node performs a local search among its neighbors: if a packet's destination is found in its nearest neighborhood, it will be delivered to the target. Otherwise, it is randomly forwarded to one of the neighbors of the searching node. We select these two strategies to represent the sets of routing strategy: routing with only local information and routing with system-wide information.

The fundamental diagram of network traffic is investigated. Fundamental diagram (flux-density relation) is one of the important criteria that evaluates the transit capacity for a traffic system. To maintain a constant density traffic, a new packet is added to the system whenever a packet is removed. The flux is recorded as the number of packets successfully arrived at destinations per time step.

We firstly present the simulation results with localsearching strategy. Figure.1 shows the simulation results on scale-free networks. In Fig.1(a), one can see that the flux suddenly drops to a very small value at a density threshold of $\rho_{1}$. The system changes from free flow state to congested state. When decreasing the packet density, the system remains in congested state until a second threshold $\rho_{2}<\rho_{1}$ is reached. Thus the hysteresis is found between $\rho_{2}$ and $\rho_{1}$, indicating that the system is bistable in a range of packet density. In order to explain this phenomenon, we investigate the number of packets delivered from one node to other node per time step $N_{p-s e n t}$, and the number of jammed nodes $N_{n-\text { jam }}$, as shown in 


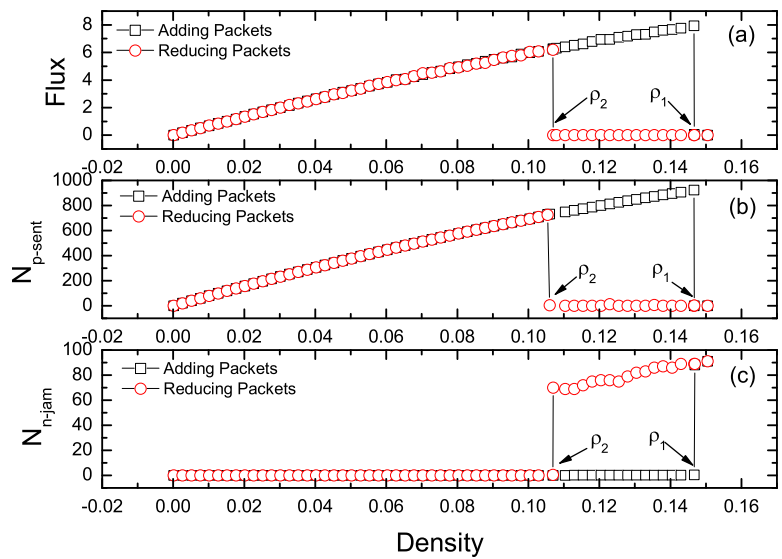

FIG. 1: (Color online). (a) Flux-density relation on scale-free networks using local searching strategy. (b) The number of packet delivered from one node to other node per time step $\left(N_{p-\text { sent }}\right)$ vs density. (c) Number of congested nodes $\left(N_{n-j a m}\right)$ vs density. Node capacity parameters are $\alpha=1.0$ and $\beta=0.3$.

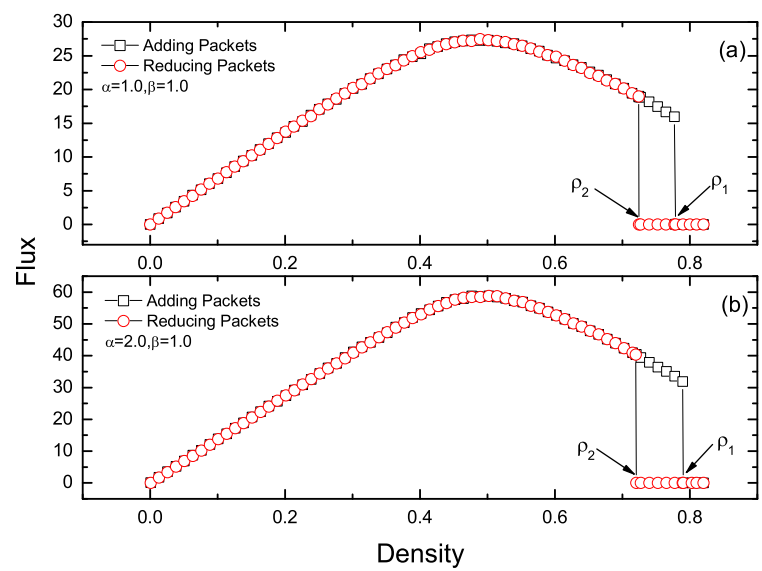

FIG. 2: (Color online) Flux-density relation on scale-free networks using local searching strategy with node capacity parameters: (a) $\alpha=1.0, \beta=1.0$; (b) $\alpha=2.0, \beta=1.0$.

Fig.1(b) and Fig.1(c). A node is jammed when the number of packets in it reaches its queue length $L$. One can see that at $\rho_{1}$, $N_{p-\text { sent }}$ decreases and $N_{n-\text { jam }}$ increases suddenly. That is, only very few packets can be delivered because most nodes are congested. Because Flux $\sim N_{p-\text { sent }}$, and Flux $\sim \frac{1}{N_{n-j a m}}$, it is easy to understand that Flux will become small above $\rho_{1}$. When decreasing the density from a congested state, $N_{n-\text { jam }}$ remains high and $N_{p-s e n t}$ remains low until $\rho_{2}$ is reached. Thus the system remains in congested state between $\rho_{2}$ and $\rho_{1}$. This is in agreement with the result of Fig.1(a).

Figure 2 shows the simulation results of the special case of

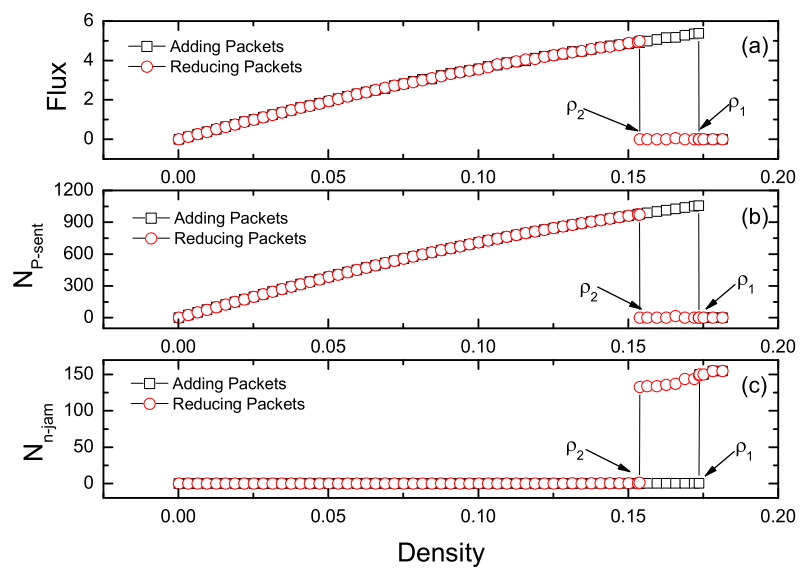

FIG. 3: (Color online) (a) Flux-density relation on small-world networks using local searching strategy with node parameters $\alpha=1.0$ and $\beta=0.3$. (b) $N_{p-\text { sent }}$ vs density. (c) $N_{n-j a m}$ vs density.

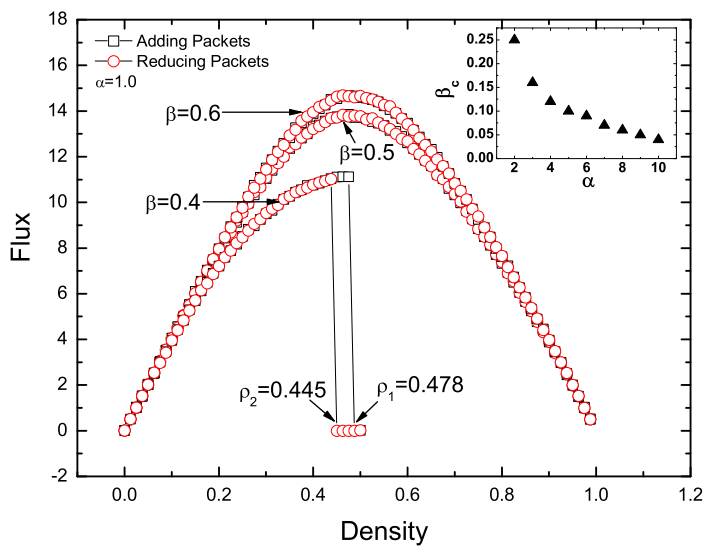

FIG. 4: (Color online) Flux-density relation on small-world networks using local searching strategy with node parameters $\alpha=1.0$ and different values of $\beta$. The inset shows the variation of threshold value $\beta_{c}$ with different values of $\alpha$.

$\beta=1$, that is, the handling ability is equal to the length of queue, so every packet in the queue can be delivered in one step as long as the surrounding nodes are not congested. The hysteresis persists with larger value of $\alpha$, as shown in Fig.2(b). Moreover, the values of $\rho_{1}$ and $\rho_{2}$ are much larger than those in Fig.1. Other simulations also show that the values of $\rho_{1}$ and $\rho_{2}$ will increase with $\beta$. This indicates that the system can maintain a high flux state with very high packet density when the delivery ability of nodes are large enough.

In small-world networks, as shown in Fig.3, hysteresis happens in the flux-density relation when $\alpha=1.0$ and $\beta=0.3$. When the value of $\beta$ increases, the hysteresis loop will dis- 

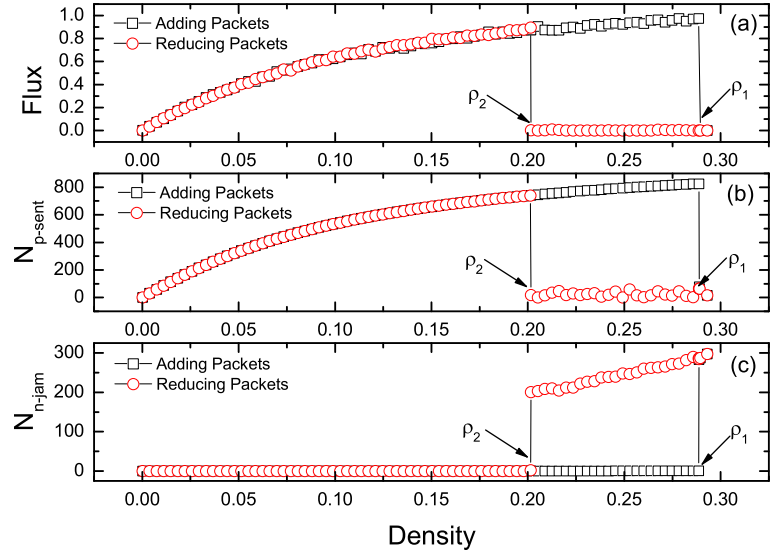

FIG. 5: (Color online) Flux, $N_{p-\text { sent }}$ and $N_{n-\text { jam }}$ vs density on lattice grid using local searching strategy with node parameters $\alpha=1.0$ and $\beta=0.2$.

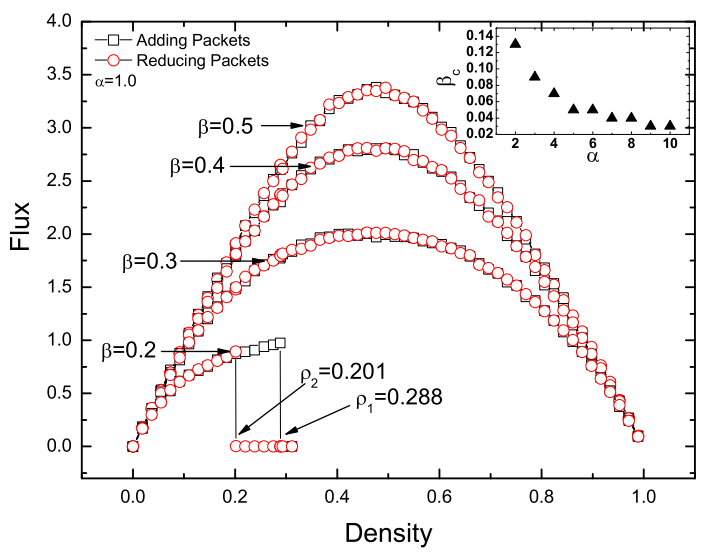

FIG. 6: (Color online) Flux-density relation on lattice grids using local searching strategy with node parameters $\alpha=1.0$ and different $\beta$. The inset shows the threshold of $\beta_{c}$ corresponding to different values of $\alpha$.

appear, as shown in Fig.4. When $\beta \geq 0.5$, the curves of increasing and decreasing density overlap. Thus $\beta_{c}=0.5$ is the threshold above which the hysteresis disappears. This behavior is different from that of the scale-free networks, where the phenomenon of hysteresis persists for different node parameters. We find that the threshold value of $\beta_{c}$ changes for different values of $\alpha$. The variation of $\beta_{c}$ with $\alpha$ is shown in the inset of Fig.4. Generally, $\beta_{c}$ decreases with $\alpha$.

Figure 5 shows the flux-density relation on lattice grids with local searching strategy. Flux, $N_{p-\text { sent }}$ and $N_{n-j a m}$ as a function of network's packet density are shown. In Fig.6, one can see that the phenomenon of hysteresis exists for low $\beta$ values, but it vanishes when $\beta$ increases above a threshold. When

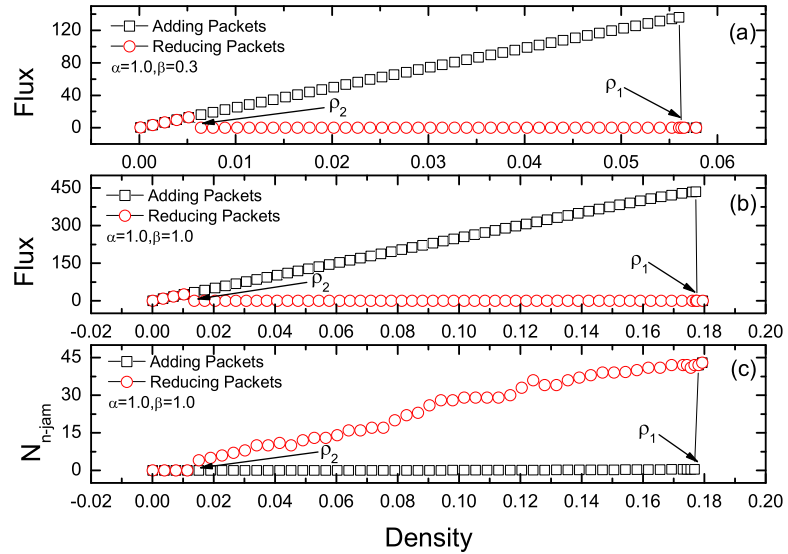

FIG. 7: (Color online) Flux-density relation for the scale-free networks using shortest path strategy: (a) $\alpha=1.0, \beta=0.3$. (b) $\alpha=1.0$, $\beta=1.0$. (c) Variation of $N_{n-\text { jam }}$ as a function of network's density with $\alpha=1.0, \beta=1.0$.

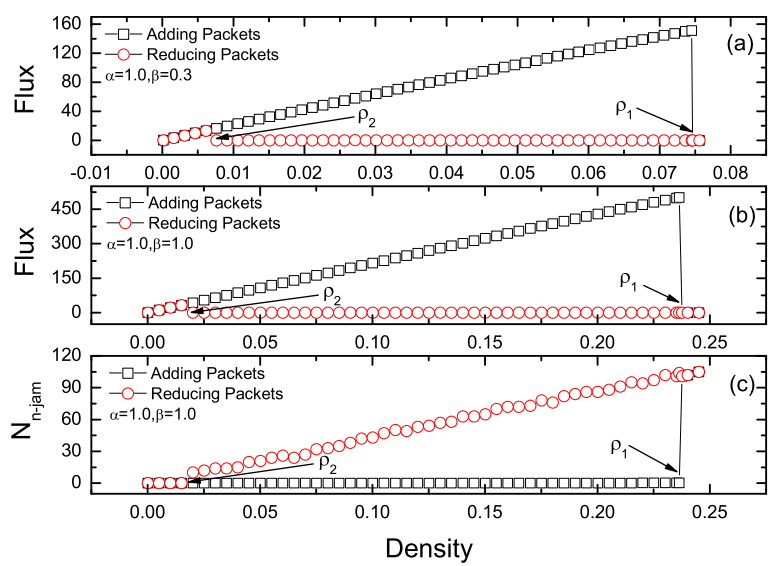

FIG. 8: (Color online) Flux-density relation for the small-world networks using the shortest path strategy. (a) $\alpha=1.0, \beta=0.3$. (b) $\alpha=1.0, \beta=1.0$. (c) Variation of $N_{n-j a m}$ as a function of network's density with $\alpha=1.0, \beta=1.0$.

$\beta \geq 0.3$, the hysteresis disappears. The behavior is similar to that of small-world networks. The value of $\beta_{c}$ for the hysteresis to disappear is also sought out for different values of $\alpha$, as shown in the inset of Fig.6. Again, one can see that $\beta_{c}$ decreases with $\alpha$.

Now we present the simulation results of the shortest path strategy on different networks. In Fig.7, the simulation results of scale-free network are shown. One can see that the hysteresis persists with scale-free networks, whether the local searching strategy or the shortest path strategy is employed. Comparing Fig.1(a) and Fig.7(a), the flux of the shortest path 


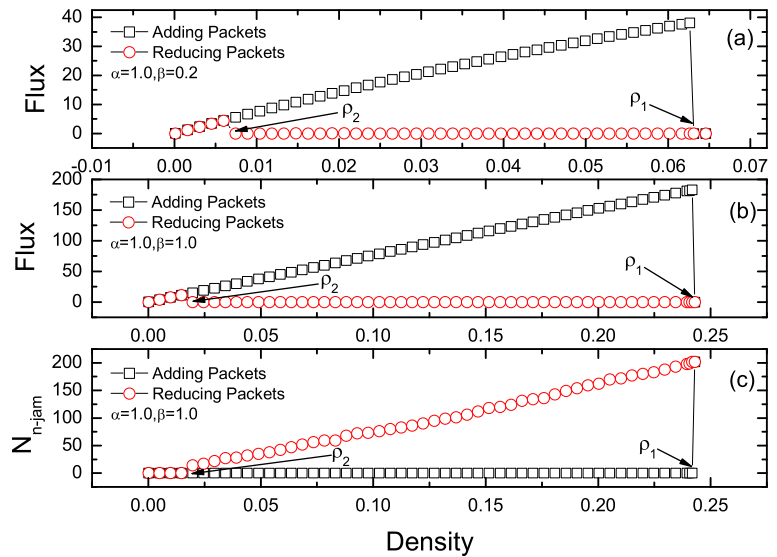

FIG. 9: (Color online) Flux-density for the lattice networks using the shortest path strategy: (a) $\alpha=1.0, \beta=0.2$. (b) $\alpha=1.0, \beta=1.0$. (c) Variation of $N_{n-\text { jam }}$ as a function of network's density with $\alpha=1.0$, $\beta=1.0$.

strategy in the free flow state is much larger than that of the local searching strategy for the same density. However, with the shortest path strategy, the system enters the congested state at a very low packet density. The values of $\rho_{1}$ and $\rho_{2}$ corresponding to hysteresis are far smaller than those of the local searching strategy. The shortest path strategy is easier to congest for the system.

We can define a normalized size of hysteresis loop: $\rho_{\text {loop }}=$ $\frac{\rho_{1}-\rho_{2}}{\rho_{1}}$. In the results of scale-free networks, we find that $\rho_{\text {loop }} \approx$ 0.9 for the shortest path strategy, while $\rho_{\text {loop }} \approx 0.2$ for the local routing strategy. This implies that it is more difficult for the system to recover from congested state when the shortest path strategy is employed. This is because some nodes with high betweenness are very easy to congest with the shortest path strategy. The congestion is difficult to dissipate by randomly removing packets from the system. The special case of $C=$ $L$ is also investigated, as shown in Fig.7(b). The hysteresis persists in this case. The variation of the number of congested nodes is shown in Fig.7(c). The system remains congested at $\rho_{2}<\rho<\rho_{1}$ because many nodes have not been dissipated.

Figure 8 shows the simulation results of small-world networks with the shortest path strategy. The hysteresis persists for different node capacity parameters. The flux of the shortest path strategy is much larger than that of the local searching strategy. But the system gets congested at a very low density. The hysteresis loop of the shortest path strategy is also much wider than that of local searching strategy, implying that it is difficult to recover the system to free flow state.

Figure 9 shows the simulation results of the shortest path strategy on the lattice grids. One can see that, with $\beta=0.2$ $(C<L)$ or $\beta=1.0(C=L)$, the hysteresis persists. As is shown in Fig.9(c), when the network's density reached $\rho_{1}$, $N_{n-j a m}$ increased rapidly. The system enters the congested state from free flow state. When the density is decreased to $\rho_{1}$, there are still many congested nodes until $\rho_{2}$ is reached. One can also see that the values of $\rho_{1}$ and $\rho_{2}$ are far smaller than that of the local searching strategy.

We note that in small-world networks and lattice grids, there is no topological hub nodes with high betweenness. However, the hysteresis persists for these networks when the shortest path strategy is employed. Although the small-world networks have the character of short average distance, the system is easy to congest and difficult to recover free flow state.

In summary, the flux-density relation of network traffic has been investigated in detail to evaluate the efficiency and robustness of routing strategies and underlying network structures. The phase transition and hysteresis are produced for three different networks and two routing strategies. When the local routing strategy is employed, the hysteresis only persists for the scale-free networks. When the shortest path strategy is employed, the hysteresis persists for all three network structures. Although the flux is larger than that of local searching strategy, the system gets congested at much lower density, and it is difficult to recover it to free flow state. For the smallworld networks and lattice grids, there is a threshold value $\beta_{c}$ of handling capacity for the hysteresis to disappear.

This work is funded by National Basic Research Program of China (No. 2006CB705500), the NNSFC Key Project No.10532060, Project Nos.70601026, 10672160,10872194. Y.-H. Wu acknowledges the support of Australian Research Council through a Discovery Project Grant.
[1] A.L. Barabási, R. Albert, Science 286, 509 (1999).

[2] D.J. Watts, S.H. Strogatz, Nature (London) 393, 440(1998).

[3] M.E.J. Newman, D.J. Watts, Phys. Rev. E 60, 7332 (1999).

[4] M.E.J. Newman, SIAM Rev 45, 167 (2003).

[5] R. Pastor-Satorras, A. Vespignani, Evolution and Structure of the Internet: A Statistical Physics Approach (Cambridge University Press, 2004).

[6] S. Boccaletti, V. Latora, Y. Moreno et al., Phys. Rep. 424, 175 (2006).

[7] B. Tadić, S. Thurner, G. J. Rodgers, Phys. Rev. E 69, 036102 (2004); B. Kujawski, B. Tadić, G.J. Rodgers, New J. Phys. 9,
154 (2007)

[8] M.B. Hu, R. Jiang, Y.H. Wu, W.X. Wang, Q.S. Wu, Eur. Phys. J. B 63, 127 (2008).

[9] W. Li, X. Cai, Phys. Rev. E 69, 046106 (2004).

[10] M.B. Hu, W.X. Wang, R. Jiang, Q.S. Wu, Y.H. Wu, Phys. Rev. E 75, 036102 (2007).

[11] B. Tadić, G.J. Rodgers, S. Thurner, Int. J. Bifurcation and Chaos 17, 2363 (2007).

[12] R. Guimerá, A. Díaz-Guilera, F. Vega-Redondo, A. Cabrales, A. Arenas, Phys. Rev. Lett 89, 248701 (2002). 